\title{
glissements de terrain liés directement à des travaux
}

\author{
Rapport général de \\ G. Pilot \\ Adjoint au Chef du Département des Sols et Fondations \\ Laboratoire central des ponts et chaussées - Paris
}

\section{Introduction}

Les glissements de terrain se produisent sous l'action d'un (ou plusieurs) "facteur déclenchant " qui rompt l'équilibre, soit au niveau des forces massiques, soit au niveau des forces extérieures, ou au niveau des forces de liaison dans le massif.

Certains de ces facteurs déclenchants sont naturels, tels que séismes, érosions, fluctution des écoulements hydrauliques, altération, etc.

A l'échelle des travaux de génie civil, l'activité humaine s'ajoute à ces facteurs naturels et constitue fréquemment un facteur essentiel dans la rupture de l'équilibre des massifs.

Du seul point de vue du rôle de l'activité humaine le sujet à traiter est très vaste, puisqu'il couvre des matériaux très divers, des argiles molles aux massifs rocheux, et des ouvrages très variés (barrages, ports, bâtiments, infrastructures, etc.).

Face à une telle diversité il fallait se limiter à quelques aspects du problème : on a donc choisi de traiter trois sujets de mécanique des sols : les remblais sur sols mous, les talus de déblais, les constructions sur versants.

Après avoir présenté les facteurs d'instabilité on décrit les principales configurations de tels glissements induits par des travaux en insistant sur le mécanisme des ruptures. On s'est efforcé d'illustrer ces conditions par des exemples concrets ètudiés au L.C.P.C. ou puisés dans la bibliographie de ces dix dernières années. Des considérations plus détaillées, notamment en ce qui concerne les méthodes de calcul, les projets et les confortements, peuvent être trouvées dans les états des connaissances présentés aux congrès internationaux de mécanique des sols de Mexico (Skempton, Hutchinson, 1969), Moscou (Bjerrum. 1973), Tokyo (Morgenstern et al., 1977) et Stockholm (La Rochelle, Marsal, 1981).

\section{Les facteurs d'instabilité}

Les glissements de terrain se produisent le long d'une surface de glissement (fig. 1), discontinuité plus ou moins complexe, lorsque la contrainte de cisaillement $\tau$ devient supérieure à la résistance au cisaillement $\tau_{\text {max }}$. On exprime le coefficient de sécurité $F$, au moins localement, par la relation

$$
\begin{aligned}
F=\frac{\tau \max }{\tau}= & \frac{\mathrm{c}^{\prime}+\sigma^{\prime} \operatorname{tg} \phi^{\prime}}{\tau}=\frac{\mathrm{c}^{\prime}+(\sigma-\mathrm{u}) \operatorname{tg} \phi^{\prime}}{\tau} \\
& (\mathrm{F}=1 \text { à la rupture }) .
\end{aligned}
$$

L'extension de cette relation à l'ensemble de la courbe de rupture, plane, circulaire ou de forme quelconque, considérant ou non que la rupture se produit simultanément en tout point, conduit à l'expression globale du coefficient de sécurité du talus. Des formulations différentes s'emploient dans le cas d'autres mouvements de terrains tels que le fluage ou les coulées de boue.

On trouve dans cette expression les différentes grandeurs sur lesquelles agissent les facteurs d'instabilité.

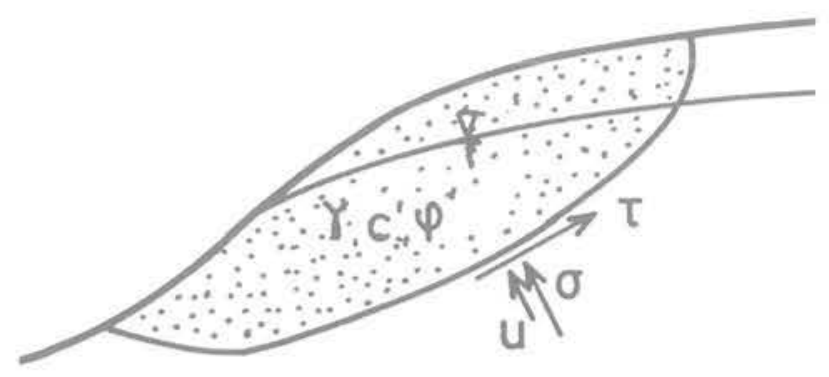

Fig. 1 Eléments de définition du coefficient de sécurité d'un talus 
Lorsque le facteur d'instabilité est une modification d'ordre géométrique (tranchée de déblai, remblais) ce sont les composantes $\sigma$ et $\tau$ de la contrainte qui sont affectées, ainsi que, dans le cas des sols fins peu perméables, la valeur $u$ de la pression interstitielle (apparition de surpressions positives ou négatives).

Lorsque le facteur d'instabilité est d'ordre hydraulique seul, sous forme d'une variation du niveau de la nappe, ou de variation de pression interstitielle dans un aquifère captif, c'est la valeur $u$ de la pression interstitielle qui commande l'évolution de la stabilité.

Le facteur d'instabilité peut également être d'ordre structurel : dans les argiles surconsolidées raides, par exemple, il existe généralement un réseau dense de microfissures: l'ouverture d'une tranchée de délai crée une diminution des contraintes et favorise l'ouverture de ces microfissures; l'infiltration d'eau et l'altération qui en résultent provoquent alors une diminution de la résistance au cisaillement (terme de cohésion effective).

La combinaison de ces trois facteurs d'instabilité, générés par les réalisations humaines, se fait dans des conditions extrêmement variables avec des influences très diverses de l'un ou l'autre des facteurs, lesquelles conduisent le coefficient de sécurité à diminuer. II est important de noter que, du fait des variations lentes et assez complexes des surpressions interstitielles, les conditions de stabilité les plus critiques (éventuellement la rupture) peuvent n'apparaitre qu'à long terme, bien après la fín des travaux (cas des tranchées de déblais dans les sols surconsolidés); il s'agit d'une conséquence directe de l'activité humaine et non d'un phénomène naturel, ainsi que le délai d'apparition d'une telle rupture pourrait le laisser penser.

Afin de mieux préciser les interventions respectives des divers facteurs présentés, on examine ci-dessous trois grands types "d'ouvrages en terre" particulièrement menacés par les glissements de terrain; remblais sur sols mous, talus de déblais, construction sur versant.

\section{Remblais sur argiles molles}

Les constructions sur argiles molles sont assez variées : remblais, stocks de pondéreux, réservoirs à hydrocarbures et autres produits chimiques, silos à grains et autres produits pulvérulents. Leurs comportements varient selon la rigidité de l'ouvrage construit, c'est-à-dire selon l'adaptation de la structure aux déformations du sol.

Le mécanisme de la rupture, poinçonnement vertical ou rupture rotationnelle (fig. 2) est donc variable en fonction du sol et de la structure, mais la mobilisation de la résistance au cisaillement est identique dans les différents cas, en sorte qu'on se limitera à l'examen d'un type d'ouvrage: on a choisi le cas des remblais parce qu'ils ont fait l'ojet des travaux et synthèses les plus nombreux (Bjerrum 1972, 1973, Pilot 1976. Tavenas 1980).

Lors de la construction d'un tel ouvrage sur argile molle, les contraintes totales dans le massif augmentent, de même que la pression interstitielle de l'eau. Les calculs montrent, et les constatations confirment, qu'il en résulte une diminution du coefficient de sécurité en sorte que lorsque des glissements se produisent, c'est essentiellement pendant la phase de construction (rupture à court terme). Ces ruptures sont sensiblement circulaires et profondes, passant largement sous
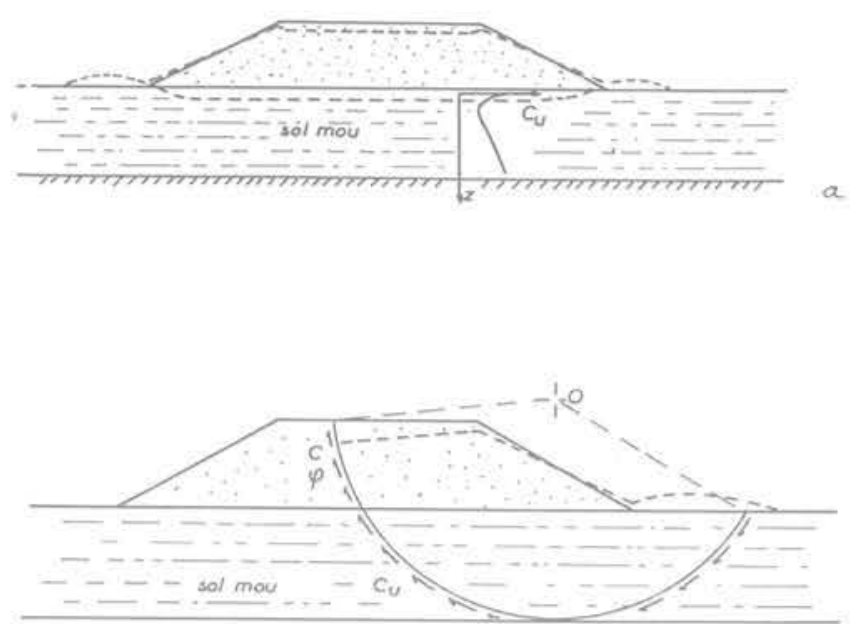

Fig. 2 Modes de rupture d'un remblai construit sur argile molle

a) Poinçonnement

b) Rupture rotationnelle

le talus du remblai. A l'issue de la construction les contraintes totales demeurant constantes et la pression interstitielle décroissant, du fait de la consolidation, le coefficient de sécurité augmente.

Ce schéma n'est mis en défaut, lors de ruptures différées, que dans de rares cas de sols à anisotropie fortement marquée : il se produit un transfert horizontal d'eau interstitielle et une augmentation des pressions interstitielles sous les talus du remblai, situation dont peut résulter une situation temporaire de faible valeur du coefficient de sécurité.

Ce sont donc les surpressions interstitielles résultant du chargement qui gouvernent la stabilité; leur détermination a priori demeure difficile dans la mesure où les relations théoriques ou semi expérimentales $\Delta u=f\left(\bar{\sigma}_{i}\right)$ n'ont pas reçu de confirmation par les mesures réalisées en vraie grandeur.

Par contre, la synthèse de ces mesures (Leroueil et al., 1978) a permis de préciser l'évolution de $\Delta u$, dans l'axe d'un remblai, en fonction de l'augmentation $\Delta \sigma_{v}$ de la contrainte verticale (fig. 3 ); on distingue les trois phases suivantes (fig. 4):

- au début du chargement, tant que $\Delta \sigma_{v}$ est inférieur à la pression de préconsolidation $\sigma_{\mathrm{p}}^{\prime}, \Delta \mathrm{u}$ varie moins vite que $\Delta \sigma_{v}\left(B_{1}=\frac{\Delta u}{\Delta \sigma_{v}}<1\right)$, ceci étant dû aux effets tels que la non saturation, la structure de l'argile, etc;

- par la suite, $\Delta \mathrm{u}=\Delta \sigma_{\mathrm{v}}\left(\overline{\mathrm{B}_{1}}=\frac{\Delta \mathrm{u}}{\Delta \sigma_{\mathrm{v}}}=1\right)$;

- enfin, lorsque le processus de rupture s'amorce, puis se développe, $\Delta u$ croît plus vite de $\Delta \sigma_{v}$

$$
\left(\bar{B}_{1}=\frac{\Delta u}{\Delta \sigma_{v}}>1\right) \text {. }
$$

Les pressions interstitielles mesurées permettent de faire des calculs de stabilité, en cours de construction, en cntraintes effectives (Pilot et al., 1982); la quasi impossibilité de leur prévision amène à faire les calculs de stabilité prévisionnels en contraintes totales sur la base de la cohésion non drainée $\mathrm{c}_{u}$.

L'évaluation de la stabilité, au stade des projets, se fait donc à partir de ces valeurs $c_{u}$, généralement mesurées au scissomètre du chantier. 


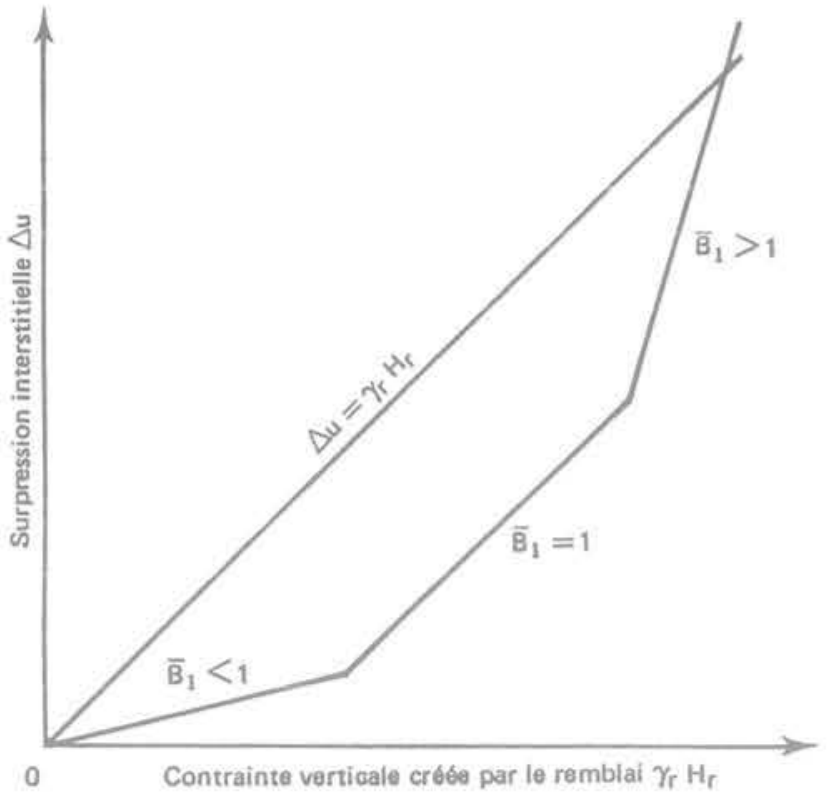

Fig. 3 Evolution des surpressions interstitielles dans une argile molle en fonction de l'augmentation de la contrainte verticale dans l'axe d'un remblai

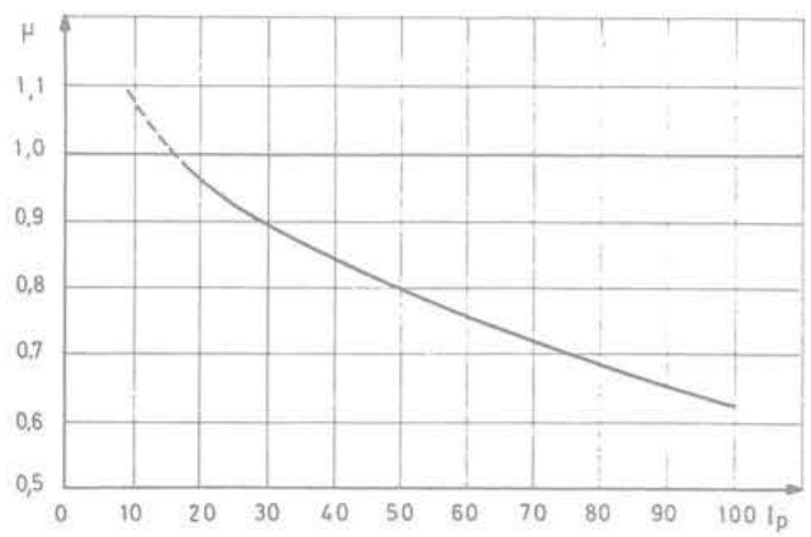

Fig. 4 Coefficient correcteur $\mu$ (IP) de la cohésion non drainée $c_{u}$ des argiles molles sous remblai (Bjerrum, 1972)

On notera cependant que, lors de la mesure au scissomètre, les effets de l'anisotropie du sol de fondation et de la vitesse de cisaillement ne sont pas sollicités dans les mêmes conditions que lors de la rupture d'un remblai; il est donc nécessaire d'opérer une correction par le biais du coefficient $\mu$ de Bjerrum, directement lié à l'indice de plasticité IP du sol (Bjerrum, 1972).

$$
\mathrm{C}_{\mathrm{U}_{\text {sous tembiai }}}=\mathrm{C}_{u_{\text {seissamiore }}} \times \mu(\mathrm{IP}) \text {. }
$$

Ce coefficient $\mu$ est donné en fonction de IP à la figure 4 .

\section{Talus de déblais}

Les talus de déblais se réalisent dans des conditions très variées :

- au plan du projet, les exigences du constructeur amènent à concevoir :

- soit des talus dont la stabilité doit être assurée tant à court qu'à long terme : c'est le cas des infrastructures de transport (autoroutes, voies ferrées...);
- soit des talus dont on ne se préoccupe que de la stabilité à court terme : fouilles de certains bâtiments, par exemple;

- soit des talus dont on s'accommoded'une stabilité précaire : cas des exploitations minières:

- au plan des sols, les comportements sont assez sensiblement différents selon qu'il s'agit d'argiles molles, d'argiles raides fissurées, de massifs argileux discontinus, etc.

On commence donc par expliciter ci-après le mécanisme du comportement à court et à long terme dans l'argile, puis on examine d'une façon concrète la stabilité des talus dans les argiles molles, dans les argiles raides, dans les argiles fissurées et on termine par deux illustrations concernant le secteur bâtiment, d'une part, celui des mines, d'autre part.

\subsection{Stabilité à court terme et stabilité à long} terme des massifs argileux

La question se pose de savoir si la période critique de stabilité d'un talus de déblai donné sera à court terme (en cours ou en fin de construction) ou à long terme (éventuellement plusieurs années après la réalisation). La réponse à cette question réside dans la valeur atteinte par les pressions interstitielles en fin de construction et dans leur évolution ultérieure (régime hydraulique transitoire qui tend vers le régime permanent déterminé par les conditions aux limites).

L'évolution des pressions interstitielles, ainsi que de la stabilité correspondante, ont étè très clairement illustrées par Bishop et Bjerrum en 1960.

Sur la figure $5 \mathrm{a}$, on a représenté un talus de déblai, assorti des positions initiales et finales de la nappe, une courbe potentielle de rupture et un point particulier où on examine l'évolution de la pression interstitielle.

En ce point, la surpression interstitielle $\Delta \mathrm{u}$ atteinte en fin de construction dépend de la variation des contraintes, de la durée de la construction et de la nature du sol.

Une valeur théorique de $\Delta u$ est obtenue par application de la classique formule de Skempton

$$
\Delta u=B\left|\Delta \sigma_{3}+A\left(\Delta \sigma_{1}-\Delta \sigma_{3}\right)\right| \text {. }
$$

Des ordres de grandeur de $A$ sont 0 et 1 respectivement pour une argile raide surconsolidée et une argile molle normalement consolidée. La figure $5 \mathrm{~b}$ indique clairement les différences de pressions interstitielles régnant dans le massif selon l'état de consolidation de l'argile. Pendant la construction, rapide, de la tranchée, le coefficient de sécurité global du talus décroît rapidement, l'allure de sa variation demeurant cependant liée à l'état du sol (fig. $5 \mathrm{c}$ ).

Durant la période de redistribution de la pression interstitielle, le coefficient de sécurité global continue à décroître sensiblement dans le cas de l'argile raide $(A=0)$, légèrement dans le cas de l'argile molle. $A$ l'équilibre des pressions interstitielles (long terme), en admettant un régime hydraulique et des paramètres effectifs de résistance au cisaillement identiques dans les deux types de sol, une valeur unique du coefficient de sécurité global est atteinte.

De part les positions des courbes de variation du coefficient de sécurité (fig. $5 \mathrm{c}$ ), on imagine bien que si la valeur finale de $\mathrm{F}$ était inférieure à 1 , la rupture serait obtenue dans l'argile molle $(A=1)$, soit pendant la construction, soit peu après son achèvement, alors que 

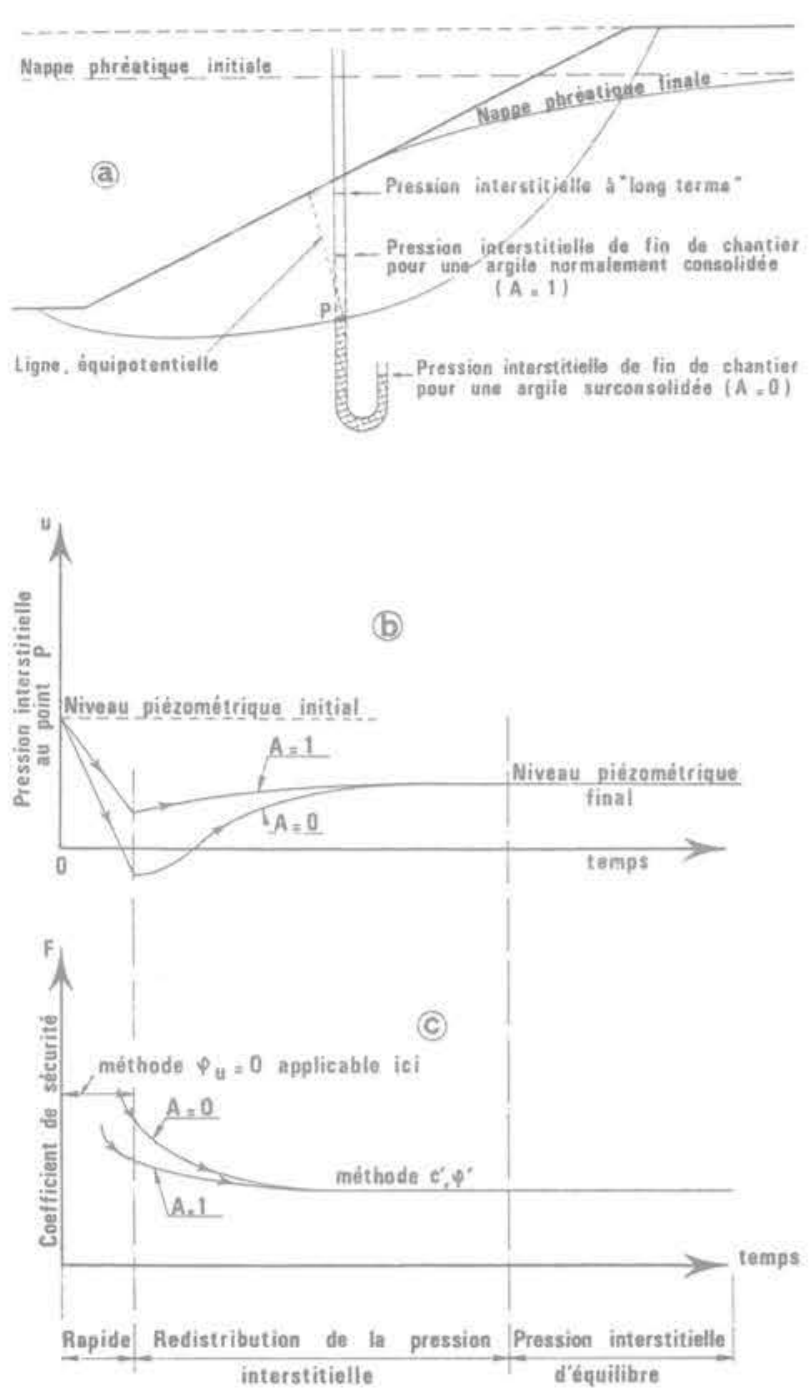

Fig. 5 Variation de la pression interstitielle et évolution du coefficient de sécurité dans un talus de déblai argileux (Bishop et Bjerrum, 1960)

pour largile raide $(A=0)$, cette rupture se manifesterait bien après la fin de construction.

Cette schématisation concorde bien avec l'observation courante que les ruptures de talus de déblai se produisent à court terme dans les sols normalement consolidés ou légèrement surconsolidés, tandis que des ruptures à long terme s'observent dans les sols fortement surconsolidés.

Des exemples sont fournis dans les deux chapitres qui suivent.

\subsection{Talus de déblai dans des argiles molles}

Un certain nombre de ruptures a été observé et étudié en détail dans des argiles molles; on prendra pour exemple la rupture expérimentale provoquée près de Bordeaux, à Bosse-Galin (Blondeau, Queyroi, 1975).

Le sol de fondation consiste en une argile organique reposant à $10 \mathrm{~m}$ de profondeur sur un sable limoneux; sa teneur en eau est de l'ordre de $60 \%$ et ses limites d'Atterberg respectivement de $40 \%$ et $80 \%$ environ pour la limite de plasticité et la limite de liquidité (IP de l'ordre de 40). Ce matériau possède une surconsolidation de 40 à $60 \mathrm{kPa}$, tandis que sa résistance au cisaillement non drainée $c_{u}$ mesurée au scissomètre de chantier varie entre 20 et $40 \mathrm{kPa}$.
La fouille expérimentale faisait $45 \mathrm{~m}$ de longueur et $20 \mathrm{~m}$ de largeur, tandis que la profondeur, déterminée d'après les calculs à la rupture en contraintes totales, exécutées préalablement, aurait dû atteindre $5,5 \mathrm{~m}$. La figure 6 montre que trois pentes de talus différentes avaient été choisies afin de provoquer des ruptures graduelles. Une forte instrumentation avait été mise en place pour suivre les pressions interstitielles et les déplacements.

Les ruptures se sont effectivement produites d'abord dans la pente a $60^{\circ}$ (fig. 6), ainsi que dans les talus des petits côtés de la fouille (pentes à $45^{\circ}$ ), alors que la profondeur de la fouille n'atteignait que $4 \mathrm{~m}$ (au lieu de $5,5 \mathrm{~m}$ initialement prévus).

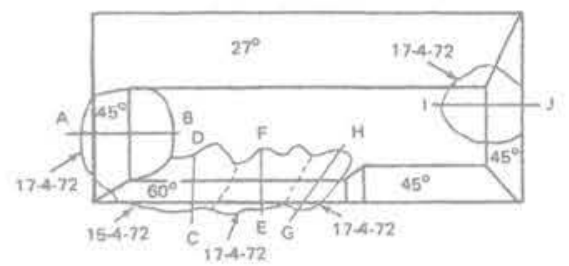

Fig. 6 Talus expérimentaux de Bosse Galin (Blondeau, Queyroi, 1972). Plan général.

C'est donc bien une rupture à court terme qui s'est produite, en accord avec le schéma présenté en 4.1. Les relevés piézométriques ont d'ailleurs indiqué une décroissance régulière de la pression interstitielle, ainsi que le montre la figure 7 .

Au plan de I'étude de stabilité, le calcul à court terme, en cntraintes totales, n'est pas satisfaisant, puisqu'à la rupture le coefficient de sécurité ressort à 1,45, au lieu de 1. La figure 8 situe le point correspondant à Bosse-Galin, ajouté dans le diagramme (F, IP) sur lequel Bjerrum (1972) avait collationné les résultats de calculs provenant de diverses ruptures de tranchées de déblai.

Ce diagramme montre que le désaccord calculobservation est comparable dans les talus de déblai en argile molle et dans les remblais sur sols mous : la correction de Bjerrum mentionnée en 3 doit donc être appliquée à la cohésion non drainée mesurée au scissomètre de chantier avant d'être introduite dans les calculs de stabilité.

On notra que dans le cas présent l'étude de stabilité en cntraintes effectives faite avec les valeurs mesurées de la pression interstitielle a donné une valeur proche de 1 du coefficient de sécurité.

\subsection{Talus de déblais dans les argiles raides surconsolidées}

On a mentionné en 4.1 que les considérations théoriques aussi bien que les observations mettaient en évidence des ruptures à long terme résultant de l'évolution spécifique de la pression interstitielle. Une question importante demeure : quel est le délai entre la fin de réalisation d'un talus de déblai et l'apparition du "long termen?

Des mesures en place assez récentes faites dans des tranchées de déblai en argile de Londres fournissent d'intéressantes données à cet égard.

On citera d'abord les résultats obtenus par Vaughan et Walbancke (1973) sur la tranchée d'Edgewarebury, taillée en 1964 dans l'argile de Londres. Sur $17 \mathrm{~m}$ de 

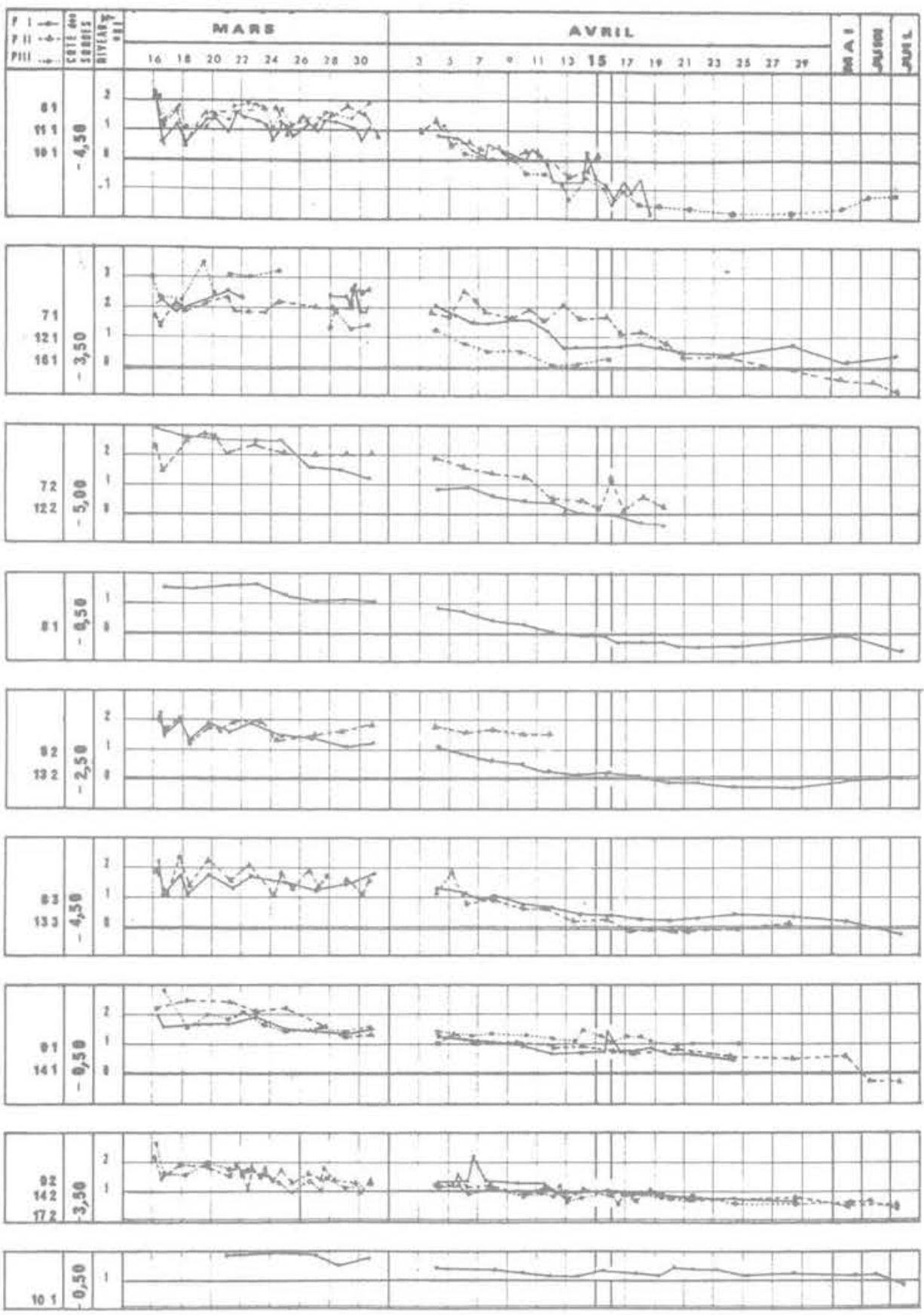

Fig. 7 Evolution de la pression interstitielle dans un talus expérimental de Bosse Galin (Blondeau, Queyroi, 1975)

hauteur, cette tranchée recoupe, à la base, l'argile bleue non altérée et au sommet l'argile brune altérée (fig. 9). Des piézomètres ont été installés en 1972 et on a observẻ que neuf ans après la réalisation de ce talus, il régnait encore de fortes pressions interstitielles négatives $(-60 \mathrm{kPa})$ assurant la stabilité de ce talus en période transitoire: les mesures effectuées en 1975 , soit 11 ans après construction, montrent que l'évolution vers le régime permanent se poursuit, mais que I'on en est encore éloigné.

Le tableau I montre cette évolution, le paramètre choisi étant la pression interstitielle réduite $r_{\mathrm{u}}$.

Des résultats plus complets ont été fournis par Walbrancke (1976) à la suite de mesures piézométriques dans la tranchée de Potters Bar, laquelle met également en jeu l'argile de Londres.
Cette tranchée de chemin de fer a été taillée en 1850 , puis élargie d'un côté en 1956 (fig. 10). Les mesures piézométriques réalisées en 1975 permettaient donc une comparaison entre un régime hydraulique "âgé " de 125 ans et un autre "âgé" de 19 ans seulement.

La comparaison des résultats se fait sur le tableau II, d'où il ressort qu'à position semblable des piézométries la pression interstitielle réduite est systématiquement sensiblement plus faible 19 ans après construction que 125 ans après.

L'ensemble des mesures de ce type a été compilé par Skempton (1977) qui a présenté l'évolution de la valeur moyenne de $r_{u}$ dans divers profils en fonction du temps. La figure 11 montre l'allure générale du phénomène: dans l'argile de Londres, il faudrait attendre une cinquantaine d'années pour que les conditions hydrauliques de la stabilité à long terme soient atteintes. 


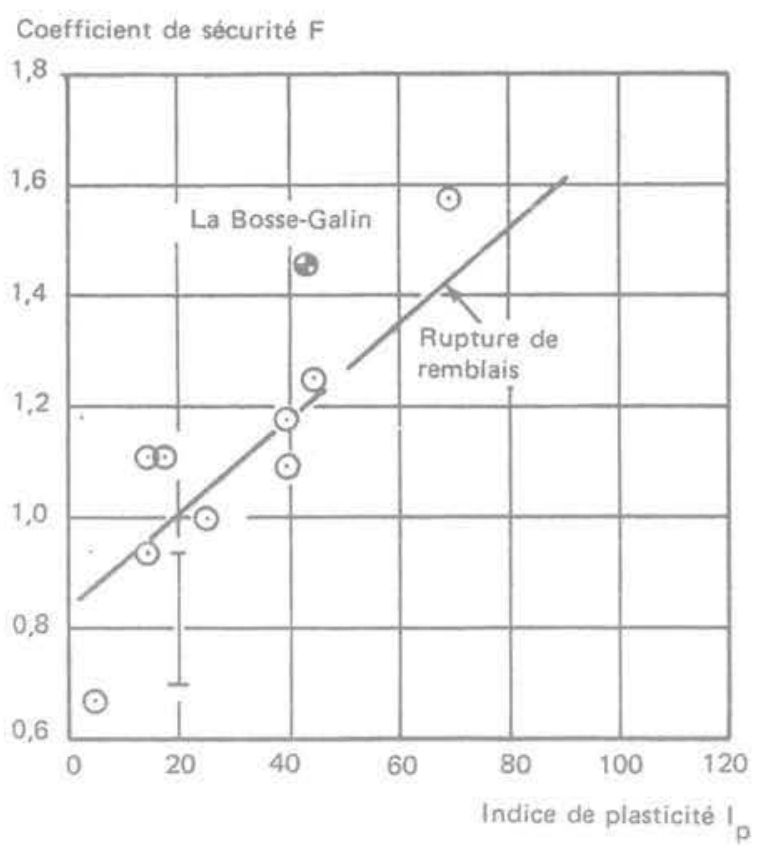

Fig. 8 Relation (F, IP) correspondant à la rupture de talus de déblais en argile molle (Bjerrum, 1973)

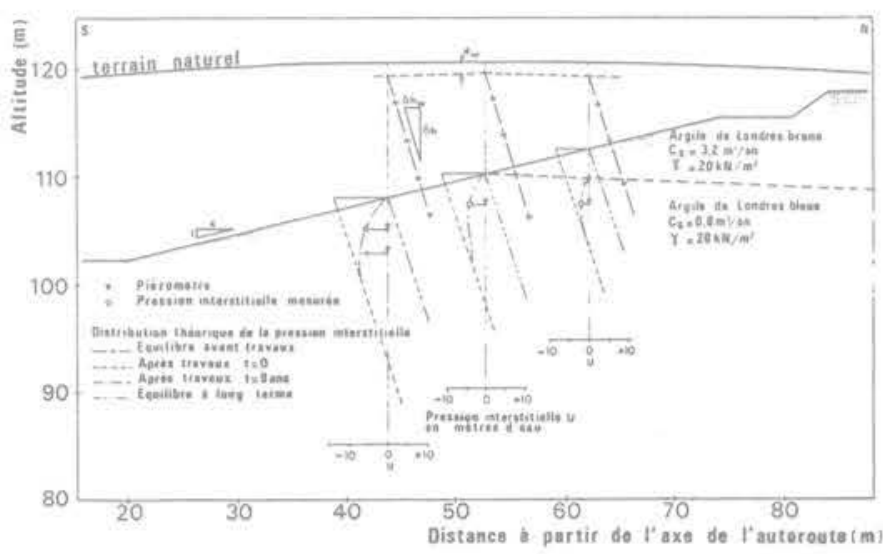

Fig. 9 Pressions interstitielles mesurées dans les talus de la tranchée d'Edgewarebury (Vaughan, Walbrancke, 1973)

Ouest

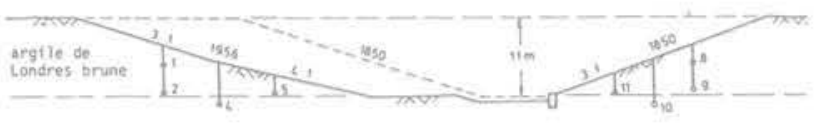

argfle de Landres bleve

Fig. 10 Pressions interstitielles mesurées dans les talus de la tranchée de Pottersbar (Walbrancke, 1976)

Ce délai ne doit pas être appliqué tel quel à toutes les formations, puisqu'il est évident que c'est la perméabilité du sol qui conditionne le délai.

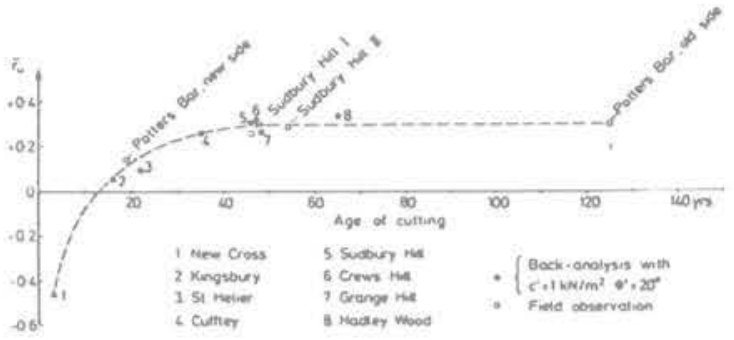

Fig. 11 Evolution des pressions interstitielles réduites, en fonction du temps, dans les talus de déblais en argile de Londres (Skempton, 1977)

On citera à cet égard deux configurations différentes :

- dans la tranchée en argile sableuse de Tronchon (Gosset, Khizardjian, 1976) les mesures piézométriques ont montré que la pression interstitielle était en relation directe avec la pluviométrie, ce qui indique que les suppressions interstitielles de construction s'étaient très rapidement dissipées.

- Dans des tranchées en argile sensible du Québec (Silvestri, 1980) il est apparu que ce matériau présentait un réseau très dense de fissures interconnectées autorisant une réponse rapide de la nappe à la pluviométrie.

L'aspect dominant de la stabilité à long terme des talus de déblai dans les argiles raides ne doit pas masquer quelques problèmes de stabilité à court terme.

Pour les raisons déjâ invoquées (impossibilité d'évaluer les pressions interstitielles) les études de stabilité à court terme sont faites en contraintes totales à partir de la cohésion non drainée $c_{u}$. Ce paramètre est généralement mesuré en laboratoire à l'appareil triaxial, sur éprouvettes de petites dimensions ( $38 \mathrm{~mm}$ de diamètre) lors d'essais rapides (de l'ordre de la dizaine de minutes).

L'expérience à montré que la résistance au cisaillement ainsi mesurée pouvait surestimer considérablement la résistance effectivement mobilisée en place. C'est ainsi que le glissement à court terme d'un talus de déblai de la fouille de la centrale nucléaire de Bradwell (Grande Bretagne) a montré que dans l'argile raide fissurée de Londres, $56 \%$ seulement de la résistance mesurée en laboratoire avait été mobilisée (Skempton, La Rochelle, 1965).

Cette surestimation résulte des effets suivants :

- effet du temps : l'essai de laboratoire, beaucoup plus rapide que la rupture en vraie grandeur, conduit à une valeur élevée de $c_{u}$ :

- effet de la fissuration : la surface de glissement en vraie grandeur chemine beaucoup plus facilement dans le réseau de fissures de l'argile que ne le fait la surface de rupture d'une petite èprouvette; il se mobilise ainsi en place une plus faible valeur de résistance au cisaillement qu'en laboratoire;

- effet de l'anisotropie : I'orientation des éprouvettes cisaillées en laboratoire (axe de l'échantillon extrait vertical) ne garantit pas que la valeur la plus faible de la résistance au cisaillement soit mise en évidence.

Ces effets se manifestent de façon extrêmement spécifique selon les argiles étudiées, en sorte qu'on ne peut donner de facteurs correctifs généraux. Le tableau III (Blivet, 1976) fournit des données relatives à quelques sols connus. 
Tableau I

Tranchée d'Edgarebury

Evolution de la pression interstitielle réduite $r_{u}$ dans le temps

\begin{tabular}{|c|c|c|}
\hline Piézomètre & $\begin{array}{c}\text { Mesure de 1973 } \\
\text { (+9 ans) }\end{array}$ & $\begin{array}{c}\text { Mesure de 1975 } \\
\text { (+11 ans) }\end{array}$ \\
\hline 1 & $-0,17$ & $+0,09$ \\
2 & $-0,22$ & $-0,32$ \\
3 & $-1,0$ & $-0,80$ \\
5 & $-1,1$ & $-0,95$ \\
6 & $-0,5$ & $-0,5$ \\
\hline
\end{tabular}

Tableau II

Lecture des piézomètres en 1975

Tranchée de Potters bar

\begin{tabular}{|c|r|r|r|r|c|}
\hline $\begin{array}{c}\text { Talus nouveau } \\
(19 \text { ans })\end{array}$ & Piéz & $r_{u}$ & Piéz & $r_{u}$ & $\begin{array}{c}\text { Talus ancien } \\
(125 \text { ans })\end{array}$ \\
\hline & 1 & 0,06 & 8 & 0,31 & \\
Moyenne & 2 & 0,18 & 9 & 0,34 & Moyenne \\
$r_{u}=0,15$ & 5 & 0,21 & 11 & 0,31 & $r_{u}=0,32$ \\
\hline
\end{tabular}

Tableau III

Effets des facteurs vitesse de déformation et fissuration

Facteurs correctifs pour la vitesse de cisaillement d'essais UU

\begin{tabular}{|c|c|c|c|}
\hline \multicolumn{2}{|c|}{ Matériau } & \multirow{2}{*}{$\begin{array}{l}\text { Facteurs correctifs } X_{1} \\
\qquad \begin{array}{c}X_{1}=\frac{C_{u}\left(t_{2}\right)}{C_{u}\left(t_{1}\right)} \\
t_{1}=10 \text { a } 15 \mathrm{~mm}\end{array}\end{array}$} & \multirow[b]{2}{*}{ Référence } \\
\hline Origine & Nature & & \\
\hline Argile de Cambridge & & $\begin{array}{c}0,91 \\
\left(t_{2}=1 i\right)\end{array}$ & \multirow[b]{2}{*}{ Skempton et Hutchinson (1969) } \\
\hline Argile de Londres & $\begin{array}{c}W_{L}=95 I_{p}=65 \\
W=33 \\
\%<2 \mu=55\end{array}$ & $\begin{array}{c}0.89 \\
\left(t_{2}=1 j\right)\end{array}$ & \\
\hline Argile plastique & & $\begin{array}{c}0,75 \text { à } 0,78 \\
\left(t_{2}=500 \mathrm{~mm}\right)\end{array}$ & \multirow[b]{2}{*}{ Wilson et Casagrande (1950) } \\
\hline Sable argileux & & 0,98 & \\
\hline Argiles des Flandres & $\begin{array}{c}W_{L}=82 I_{p}=48 \\
W=35 \\
\%<2 \mu=60\end{array}$ & $\begin{array}{c}1,15^{*} \\
\left(t_{2}=6 i\right)\end{array}$ & \multirow{4}{*}{$\begin{array}{l}\text { Article de Blondeau, Blivet } \\
\text { et Ung Seng. Résistance } \\
\text { au cisaillement des argiles } \\
\text { raides. Influence } \\
\text { des paramètres d'essais }\end{array}$} \\
\hline Argiles de Dozulé & $\begin{array}{c}W_{L}=48 I_{P}=17 \\
W=17 \\
\%<2 \mu=97\end{array}$ & $\begin{array}{c}0,65 \\
\left(t_{2}=1 j\right)\end{array}$ & \\
\hline Argiles de Provins & $\begin{array}{c}W_{L}=89 I_{p}=55 \\
W=28 \\
\%<2 \mu=95\end{array}$ & $\begin{array}{c}0,88 \\
\left(t_{2}=5 j\right)\end{array}$ & \\
\hline Argile verte & $\begin{array}{c}W_{L}=93 I_{P}=47 \\
W=32 \\
\%<2 \mu=71\end{array}$ & 1 & \\
\hline
\end{tabular}

\begin{tabular}{|c|c|c|c|c|c|}
\hline $\begin{array}{c}\text { Diamètre de l'échantillon } \\
\text { cisaillé }(\mathrm{mm})\end{array}$ & $\begin{array}{c}\text { Argile } \\
\text { de Londres }\end{array}$ & $\begin{array}{c}\text { Argile } \\
\text { verte }\end{array}$ & $\begin{array}{c}\text { Argile } \\
\text { des Flandres }\end{array}$ & $\begin{array}{c}\text { Argile } \\
\text { de Dozulé }\end{array}$ & $\begin{array}{c}\text { Argile } \\
\text { de Provins }\end{array}$ \\
\hline 16 & 1,9 & 1 & 1 & 1 & 1 \\
38 & 0,64 & 1 & 0,86 & 0,52 & 0,56 \\
153 & 0,66 & 1 & 0,5 & \\
\hline
\end{tabular}




\subsubsection{Sols résiduels}

Les sols résiduels se forment par l'altération, en place, de massifs rocheux ignés ou métamorphiques. Ce processus, illustré figure 12 (John et al., 1969), génère une structure complexe de matériaux à dominante, de haut en bas, argileuse, puis limoneuse, enfin rocheuse.

Ce mode de constitution mène à la formation de vastes réseaux de discontinuités présentant des « miroirs de glissement " et des fissures emplies de minces dépôts minéraux.

Dans tous les cas, la résistance au cisaillement le long de ces discontinuités est plus faible que dans le matériau intact. A titre d'exemple, John cite que l'angle de frottement $\phi^{\prime}$ de la matrice argileuse vaut $18,5^{\circ}$, mais s'abaisse à $14,5^{\circ}$ dans les fissures emplies d'oxides métalliques pour aller jusqu'à $10,5^{\circ}$, en présence de miroirs de glissements.

Les déblais exécutés dans ces massifs subissent des glissements complexes dont la surface est guidée par les discontinuités. La figure 13 montre la rupture d'une tranchée routière de $24 \mathrm{~m}$ de hauteur à Porto-Rico.

\subsubsection{Discontinuités d'origine glaciaire}

La présence et l'évolution de glaciers situés sur des massifs argileux s'accompagne de mouvements qui déforment l'argile jusqu'à la cisailler en son sein sur de grandes surfaces le long desquelles l'état résiduel de résistance au cisaillement peut être atteint.

L'exécution de terrassements à proximité de ces surfaces entraîne de vastes réactivations de ces mouvements anciens.

Un exemple d'une telle rupture a été clairement observé au Saskatchwan (Canada) lors de l'ouverture d'une tranchée autoroutière (Krahn et al., 1979).

La figure 14 montre le profil en travers du site; les sols comprennent une formation sablo-limoneuse de 15 à $30 \mathrm{~m}$ d'épaisseur, reposant sur 5 à $10 \mathrm{~m}$ d'argile raide dans laquelle des "miroirs de glissemen " avaient été détectés lors des sondages. Le toit de la nappe se situe à la base de la formation sablo-limoneuse.

Pour tenir compte de l'existence des discontinuités observées les calculs de stabilité avaient été exécutés en tenant compte de valeurs minimisées de résistance au cisaillement : $c^{\prime}=5 \mathrm{kPa}$ et $\phi^{\prime}=10^{\circ}$ et $15^{\circ}$, ceci conduisant à la valeur $F=1,3$ du coefficient de sécurité. En fait, la rupture se produisit dès l'achèvement de la tranchée le long d'une surface située au contact argile-limon sous-jacent.

Le calcul de stabilité “à l'envers " réalisé sur la surface de glissement observèe a montré que les valeurs des

\subsubsection{Terrassements dans des glissements exis- tants}

Des tranchées de déblai sont parfois exécutées (consciemment ou inconsciemment) dans des massifs ayant subi des glissements. L'expérience montre que le sol ne se "cicatrise" pas le long de la surface de glissement et que la résistance au cisaillement mobilisable y est abaissée au niveau de la résistance résiduelle (ce cas de figure n'est pas sans analogie avec le précédent),

paramètres de résistance au cisailiement, dans I'argile, permettant d'expliquer cette rupture sont $c^{\prime}=0$ et $\phi^{\prime}=8^{\circ}$; ces valeurs constituent la limite inférieure des

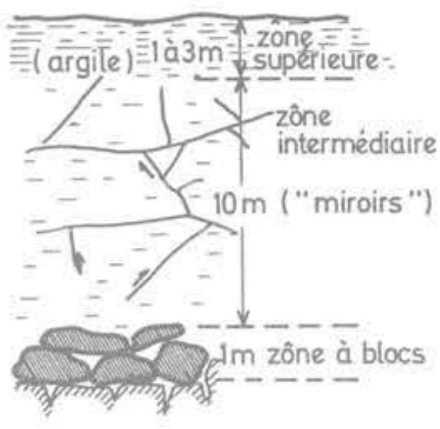

FORMATION

Fig. 12 Formation de discontinuités dans les massifs de sols résiduels (John et al., 1969)

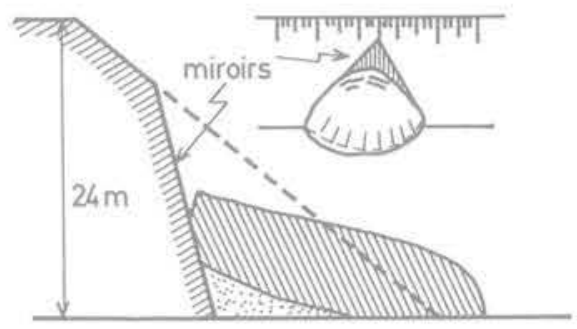

TALUS ROUTIER

Fig. 13 Glissement d'un talus de déblai dans un massif de sol résiduel (John et al., 1969)

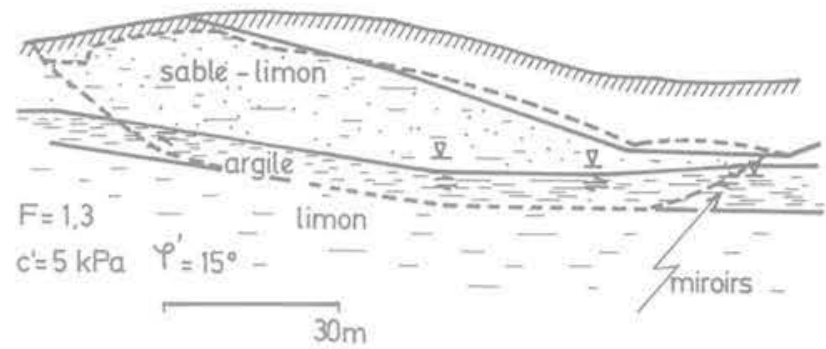

$F=1$

$\begin{array}{ll}F=1 & \varphi_{r}^{\prime}=0\end{array}$

Fig. 14 Glissement de talus le long d'une discontinuité d'origine glaciaire (Krahn, 1979)

paramètres résiduels mesurés dans les formations du crétacé de cette région.

Ce glissement s'explique ainsi par l'existence d'une surface de rupture dans la couche d'argile, surface réactivée par le déséquilibre des efforts lors du terrassement.

4.4 Talus de déblais dans les massifs argileux discontinus

Le passé géologique de certains massifs argileux se perpétue parfois sous forme de discontinuités très 


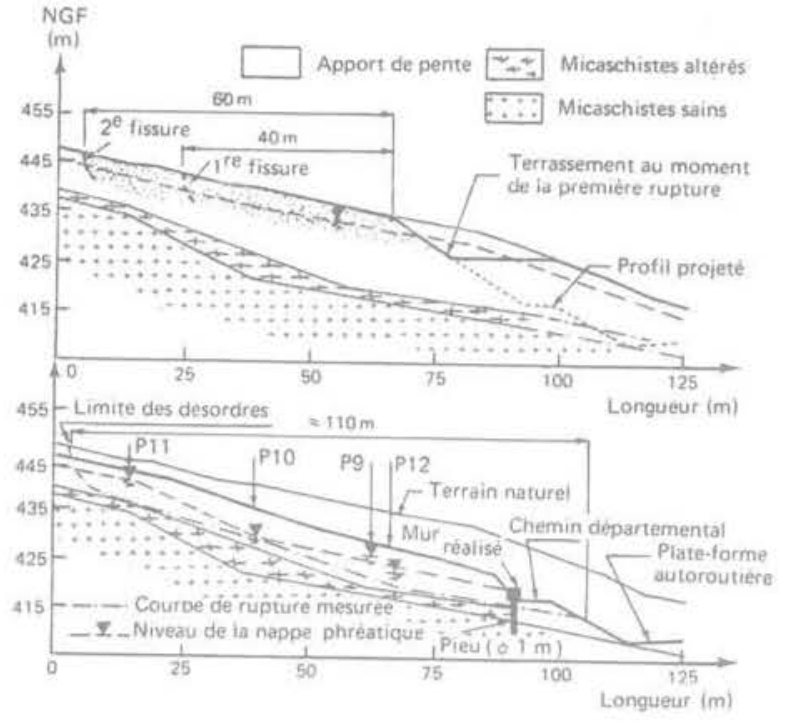

Fig. 15 Glissement de terrain réactivé par l'exécution d'un talus de déblai (Blondeau, Virollet, 1976)

étendues le long desquelles les surfaces de glissement se développent facilement; on examine ci-dessous trois cas différents de ruptures agissant dans ce contexte.

Une telle situation s'est rencontrée lors du terrassement d'un déblai autoroutier en pied de versant (Blondeau, Virollet, 1976). Les sols sont constitués (fig. 15) de $20 \mathrm{~m}$ environ d'éboulis de pente reposant sur une couche d'épaisseur métrique de micashistes altérés surmontant le micashiste sain. Le glissement s'est produit alors que le déblai atteignait environ $5 \mathrm{~m}$ de profondeur, loin encore du profil projeté; le fort développement de la zone affectée par les fissures de traction témoignait de la présence d'une rupture préexistante; ceci fut confirmé par le repérage d'un niveau de glissement dans les tubes piézométriques profonds et par le réexamen de la géomorphologie locale, laquelle révélait la présence d'un vaste mouvement ancien.
La réalisation du profil projeté a nécessité le terrassement partiel du glissement, la mise en place de drainages et la construction d'une rangée de pieux solidarisés par une longrine, remplacés par un soutènement massif en pied de talus après rupture des pieux lors de mouvements ultérieurs.

L'exécution de talus de déblais dans des formations instables est donc toujours extrêmement difficile et entraîne la mise en place d'ouvrages de soutènement très importants : un exemple en est fourni par Huder et Duerst (1981).

Des accidents de ce genre se rencontrent assez fréquemment lors de la réalisation de plate-formes, dans des versants, destinés à recevoir des bâtiments: les travaux de terrassement sont généralement peu importants et de faible coût par rapport à celui de l'opération immobilière ou industrielle : il attirent peu l'attention et n'impliquent que peu d'études. Silleran (1981) cite le cas de sites qui ont dû être abandonnés du fait de glissements inconsidérément réactivés (fig. 16)

\subsection{Talus de mines et carrières}

Le problème général des mines et carrières est de dresser les fronts de taille selon une pente qui satisfasse un compromis entre les contraintes suivantes :

- l'exploitation maximale des matériaux à extraire nécessite des talus à pente élevée:

- la stabilité doit être suffisante pour éviter des glissements qui perturbent l'exploitation et, par extension, affectent les constructions avoisinantes.

On citera deux exemples de glissements résultant de travaux de ce type :

- Zaruba et Mencl (1969), citent le cas d'une carrière d'argile ouverte au pied d'un versant, travaux qui déclenchaient un phénomène de rupture régressive. Se développant sur $140 \mathrm{~m}$ de profondeur, ces mouvements détériorèrent un bâtiment public qui dut subir de coûteux travaux de reprise en sous-ceuvre. tandis que l'exploitation de la mine était interrompue (fig. 17);

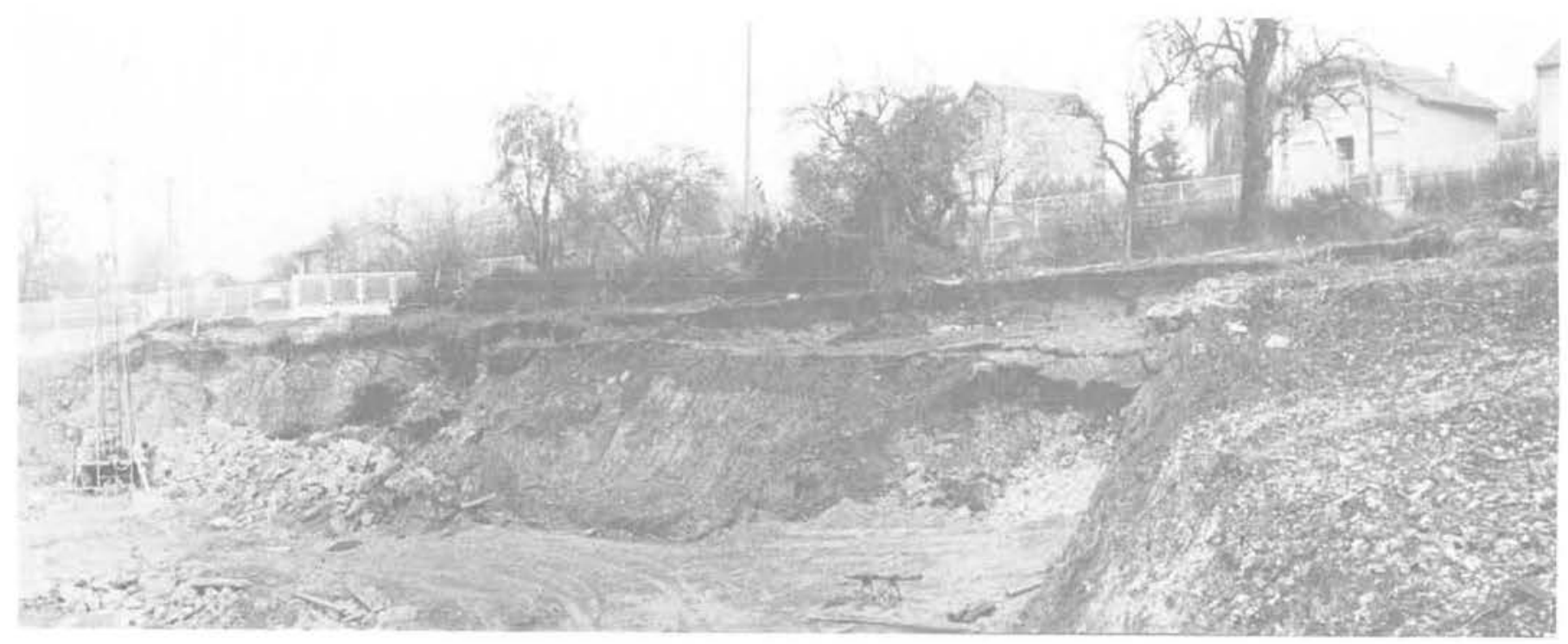

Fig. 16 Réactivation de glissements anciens à l'ouverture de fouilles de bâtiments (Silleran, 1981) 


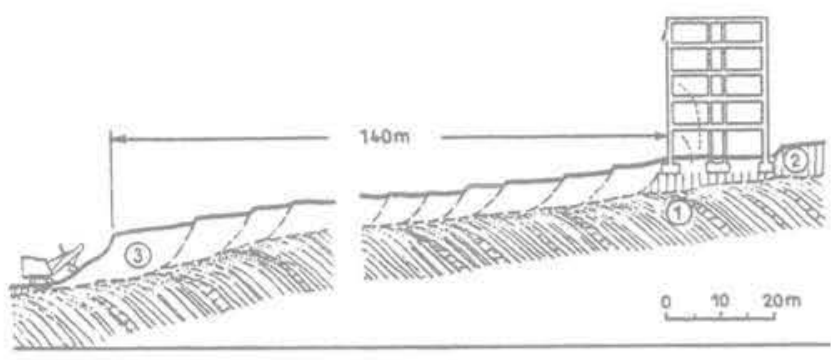

Fig. 17 Rupture régressive d'un massif argileux lors d'une exploitation de carrière (Zaruba, Mencl, 1969)

- La mine d'amiante d'Asbestos, au Québec, se présente sous forme d'un vaste puits $(2 \mathrm{~km}$ dans sa plus grande dimension) de $200 \mathrm{~m}$ environ de profondeur. (Ministère des Richesses Naturelles du Québec.)

Le massif rocheux de péridolite exploité est surmonté de sédiments meubles fluvio-glaciaires et de dépôts de lacs glaciaires comprenant aussi bien de l'argile que des bancs de sables. Cette zone de mort terrain, épaisse de plusieurs dizaines de mètres forme une couronne autour de la mine, siège de multiples instabilités. L'une des plus importantes, survenue en 1975. s'est traduite par un glissement de $500000 \mathrm{~m}^{3}$ qui s'est déversé jusqu'au fond de la mine; ce mouvement affecte une couche de 40 m d'épaisseur et s'étend sur $350 \mathrm{~m}$ (fig. 18). Les causes lointaines de ce mouvement pourraient résider dans la forte pente du talus (des désordres importants avaient commencè à se manifester plusieurs mois auparavant Le facteur déclenchant serait d'ordre hydraulique : on notera que le substratum rocheux a une forme de thalweg où un écoulement longitudinal avait été mis en évidence; en outre, au cours de l'hiver, le gel intense en surface a pu empêcher le suintement sur le talus et entraîner une élévation du niveau de la nappe. A l'appui de cette thèse, il viendrait les témoignages qui font état du dégagement initial d'une très forte quantité d'eau.

Cet incident à entraîné l'évacuation de plus de 300 personnes et la destruction de plusieurs habitations et de deux pelles de l'exploitation minière.

Le confortement a été obtenu par drainages et terrassements.

Un autre exemple intéressant de glissement de terrains de couverture induits par un exploitation minière est décrit par Bourguet et al. (1973).

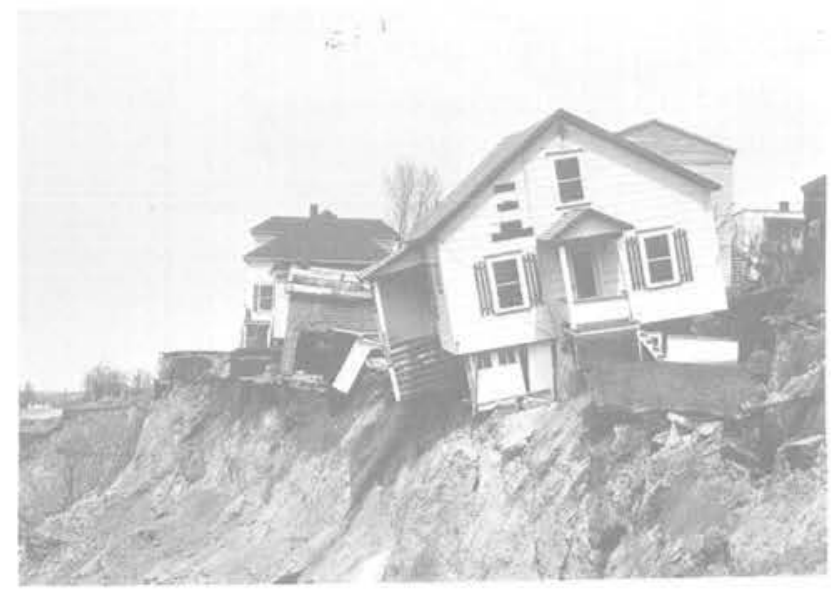

Fig. 18 Glissement d'une découverte de mine à Asbestos, Québec (Document Service Géotechnique MER)

\section{Constructions sur versants}

Les versants sont naturellement sensibles aux glissements de terrain pour de multiples raisons telles que :

- présence de nappes, éventuellement assorties d'écoulements artésiens très défavorables à la stabilité;

- formation géologique qui confère une situation d'instabilité potentielle; il en est notamment ainsi dans les conditions suivantes:

- éboulis de pente en montagne, en état permanent d'instabilité du fait des forts angles de talus;

- remplissage de thalwegs par des matériaux argileux;

- formation de couches instables dans les affleurements argileux, conséquence, par exemple, des effets périglaciaires qui ont affecté le Lias dans l'Est de la France.

Dans ces conditions, tous les travaux, même d'ampleur limitée, sont susceptibles de créer des instabilités; il en est ainsi lorsque l'on perturbe le régime hydraulique, que l'on construit des ouvrages sur ces versants et même, dans certains cas particuliers, que l'on impose des vibrations (par exemple lors du battage de pieux).

\subsection{Perturbations d'ordre hydraulique}

Parmi les perturbations d'ordre hydraulique résultant de travaux, on citera:

- abaissement des plans d'eau, lesqueis provoquent des conditions de vidange rapide. Les barrages en terre sont conçus pour résister à cet effet, mais les berges de retenues sont fréquemment affectées par des glissements importants dûs à ces abaissements : on citera la prise en compte de ce facteur dans les ètudes du barrage Idriss $1^{\text {er }}$ au Maroc (Lheriteau et al., 1977), les glissements des berges de retenue de l'Angara (Trzhtsinskii, 1978) et les déplacements des berges du réservoir de Dirillo (Italie) sous l'effet des fluctuations du niveau du plan d'eau (Japelli, Musso, 1981).

- L'infiltration des eaux de ruissellement à la suite de travaux pour lutter contre l'érosion. Les travaux de défense et de restauration des sols, très développés en Afrique du Nord visent á ralentir l'érosion des pentes, rétablir la végétation et éviter l'entraînement des sols jusque dans les réservoirs hydrauliques. A cet effet, on établit des banquettes courant le long des versants, destinées à couper, puis orienter le ruissellement. De ce fait, l'infiltration de l'eau est facilitée et il en rèsulte des écoulements internes et des glissements montrés par Jeanette et Millies-Lacroix (1965), ainsi que par Flotte (1981) qui a illustré l'apparition de ces désordres en Algérie (fig. 19).

\subsection{Construction d'ouvrages sur versants}

La construction d'ouvrages divers (bâtiments, remblais, terrils, etc.) sur versants provoque de fréquents glissements dont l'origine réside dans les facteurs suivants :

- surcharge appliquée au versant et génération de pressions interstitielles de construction:

- diminution de la perméabilité des sols sous-jacents, d'où perturbation de l'écoulement de la nappe et augmentation des pressions interstitielles; 


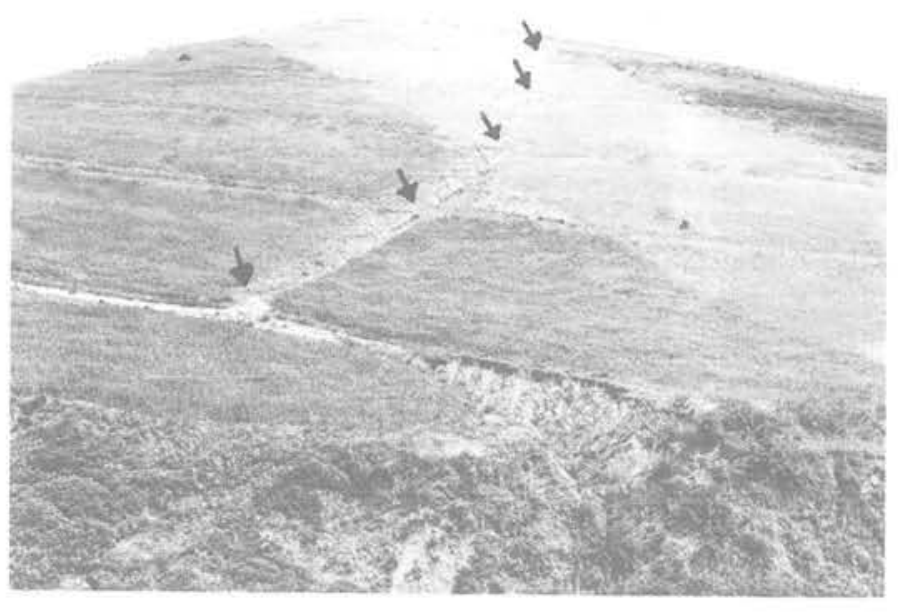

Fig. 19 Désordres dans un versant das à la création de banquettes de défense et restauration des sols (Flotte, 1981)

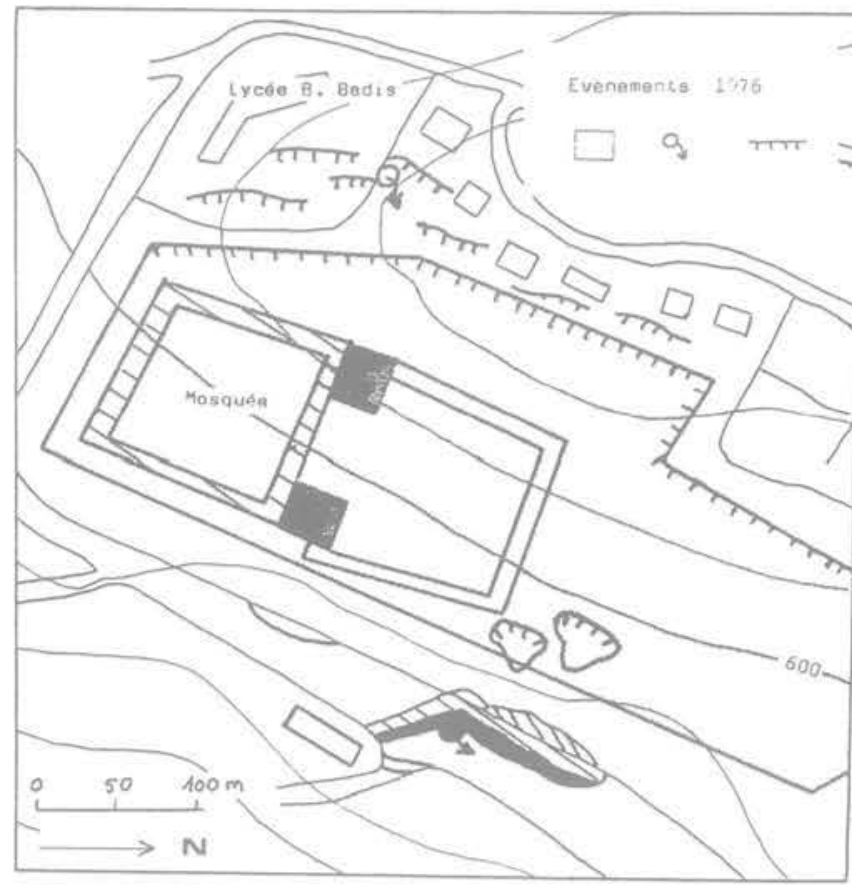

Fig. 20 Désordres observés lors de la construction de la mosquée Abd-El-Kader à Constantine (Beucler, 1981)

- Ouverture de fissures dans le versant (cas des terrils et des subsidences liées à l'exploitation souterraine) provoquant une intense infiltration et l'apparition des nappes correspondantes.

On examine ci-dessous les principaux aspects de ce problème.

\subsubsection{Désordre de bâtiments construits sur versants}

Ces désordres sont fréquents et parfois graves: les implications contencieuses qui en résultent imposent une stricte confidentialité, en sorte que peu de publications sont connues (Sanglerat, 1978); on se limitera donc à citer le glissement consécutif au travaux de la mosquée Abd-el-Khader à Constantine
(Beucler, 1981). La figure 20 montre la localisation des désordres à l'issue de cinq ans de constructions marquées par des glissements répétés :

- coupure de la route située à l'aval de la mosquée;

- rupture des conduites de gaz;

- désordres dans les infrastructures du lycée situé en amont;

- désordres dans le lotissement situé en amont;

- mise hors service du bâtiment situé en aval de la route.

Il a été constaté en permanence un accroissement des déplacements des sols de fondation et une accentuation des désordres lors de périodes de construction de la mosquée, ainsi qu'une aggravation de la situation lors des périodes pluvieuses: cela est attribué à l'infiltration facilitée par l'ouverture des fissures en amont de l'ouvrage en construction.

\subsubsection{Remblais sur versants argileux}

De nombreux versants argileux en état de stabilité précaire, voire franchement instables, sont appelés à recevoir des infrastructures, notamment routières. On sait que dans ces situations la résistance au cisaillement est réduite à l'état résiduel (Skempton, 1964), la validité de ce concept ayant été vérifiée sur sites réels (Pilot, 1969, Blondeau et al., 1977, entre autres).

La construction de remblais autoroutiers dans ces conditions a connu de nombreuses difficultés (Amar et al., 1973, Pilot et al., 1979). Ces glissements se traduisent généralement par l'affaissement d'une partie de la plate-forme et la réactivation d'une partie du glissement ancien sur lequel repose le remblai (fig. 21) : ces mouvements se produisent généralement en cours de construction, mais ils sont susceptibles d'apparaître à long terme par réactivation du glissement du versant résultant de conditions hydrauliques défavorables.

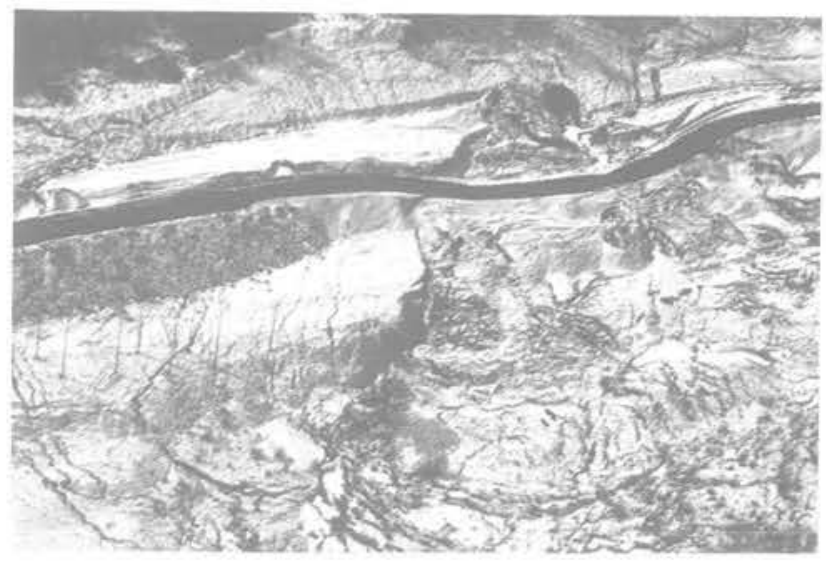

Fig. 21 Glissement d'un remblai construit sur versant instable (Document LRPC Nancy)

L'étude de tels remblais sur des zones instables demeure difficile du fait des incertitudes suivantes:

- identification de la position de la surfce de glissement;

- détermination du volume de sol susceptible de se réactiver: 


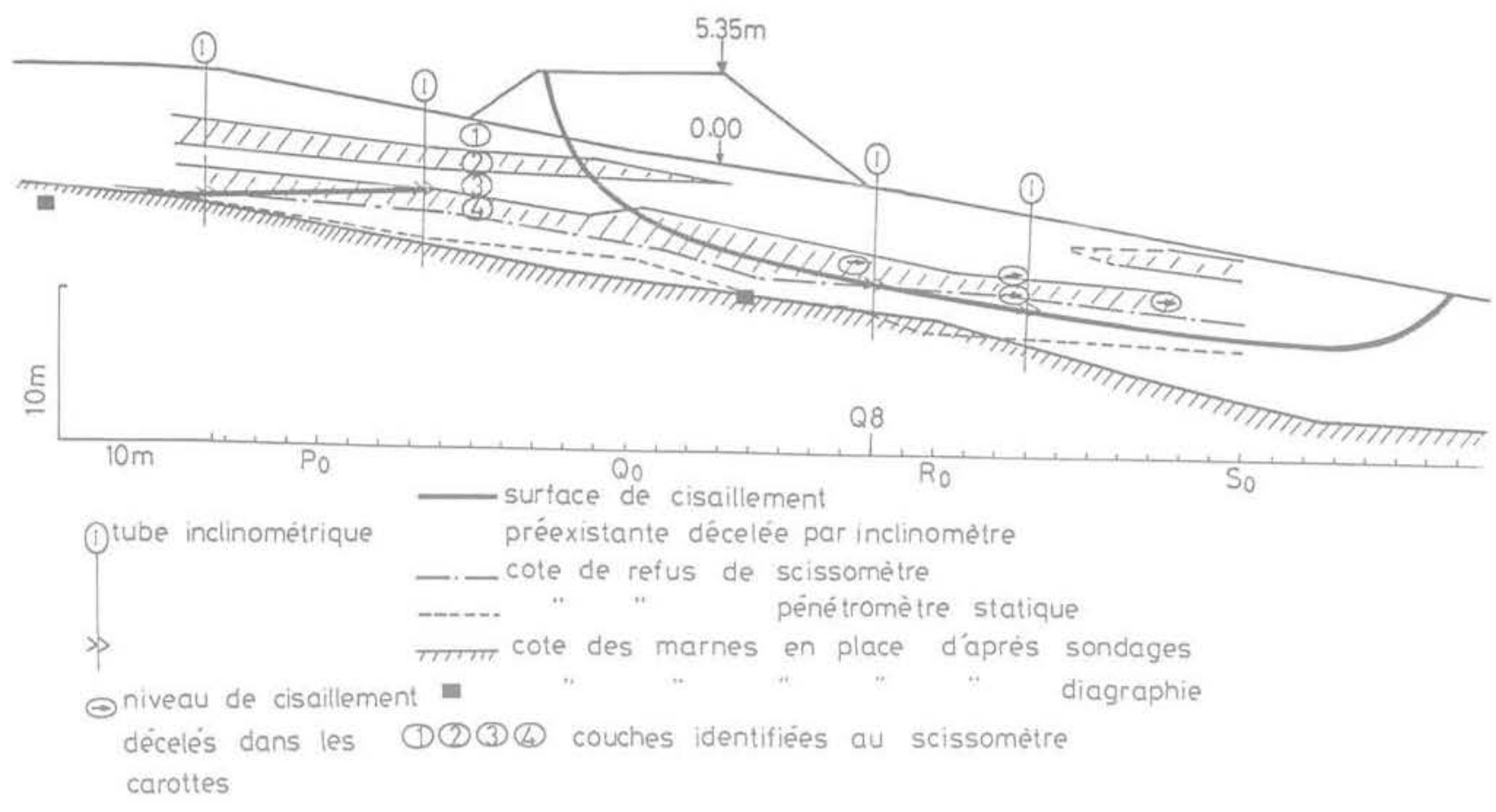

Fig. 22 Coupe des sols et du site expérimental de Sallèdes

- calcul en contraintes totales ou en contraintes effectives? Le choix se porterait a priori sur le calcul en contraintes effectives, mais il demeure le problème de l'évaluation des pressions interstitielles générées par la construction du remblai.

Ces différents aspects ont été étudiés en vraie grandeur sur le site expérimental L.C.P.C. de Sallèdes, près de Clermont-Ferrand (Blondeau et al., 1981).

Les sols de ce site (fig. 22) sont constitués de colluvions et de marnes remaniées de 7 à $10 \mathrm{~m}$ d'épaisseur reposant sur un substratum de marnes raides intactes. Une surface de rupture avait été détectée à $6.5 \mathrm{~m}$ de profondeur en moyenne.

Divers types de calculs de stabilité ont été réalisés, prenant en compte les incertitudes mentionnées ci-dessus.

Le tableau IV rassemble les résultats et montre la dispersion des valeurs obtenues. La rupture a été obtenue pour $5.35 \mathrm{~m}$ de hauteur de remblai, avec une faible augmentation de pressions interstitielles et réactivation de tout le glissement ancien situé à l'aval du glissement.

\subsubsection{Terrils de mines ou de déchets industriels}

Les terrils constituent, parmi les ouvrages humains les plus vastes et, par leur mode de mise en place, les plus sujets à subir ou déclencher des instabilités.

Parmi les accidents ayant fait l'objet de publications. on citera ceux de Rodange (Mayer, Habib, 1961) qui endommagea des routes, de Charleroi (Schrœeder, 1981), qui détruisit plusieurs immeubles et d'Aberfan (Grande-Bretagne), qui provoqua plusieurs dizaines de morts.

$\mathrm{Ce}$ dernier accident illustre l'un des caractères particuliers des glissements de terrils: les matériaux qui les constituent sont dans un état de densité lâche, très déformables, susceptibles de donner naissance à des coulées "humides" ou sèches.
Dans le cas d'Aberfan (Bishop et al., Woodland, Moore, Wardell et al., 1969) un ensemble de terrils de déchets d'exploitation charbonnière reposait sur un versant (fig. 23) principalement constitué de grès (les veines de charbon exploitées sont en profondeur), recouvertes en surface d'argile sableuse sous les terrils et d'argile à blocaux au-delà du pied du terril $n^{\circ} 7$.

C'est à la suite d'une forte période de pluie que la rupture du terril s'est produite très peu de temps après que des signes de dëformation se soient manifestés; le glissement s'est brutalement transformé en une coulée sèche qui parcouru très rapidement la pente pour achever sa course dans la ville minière d'Aberfan (fig. 24).

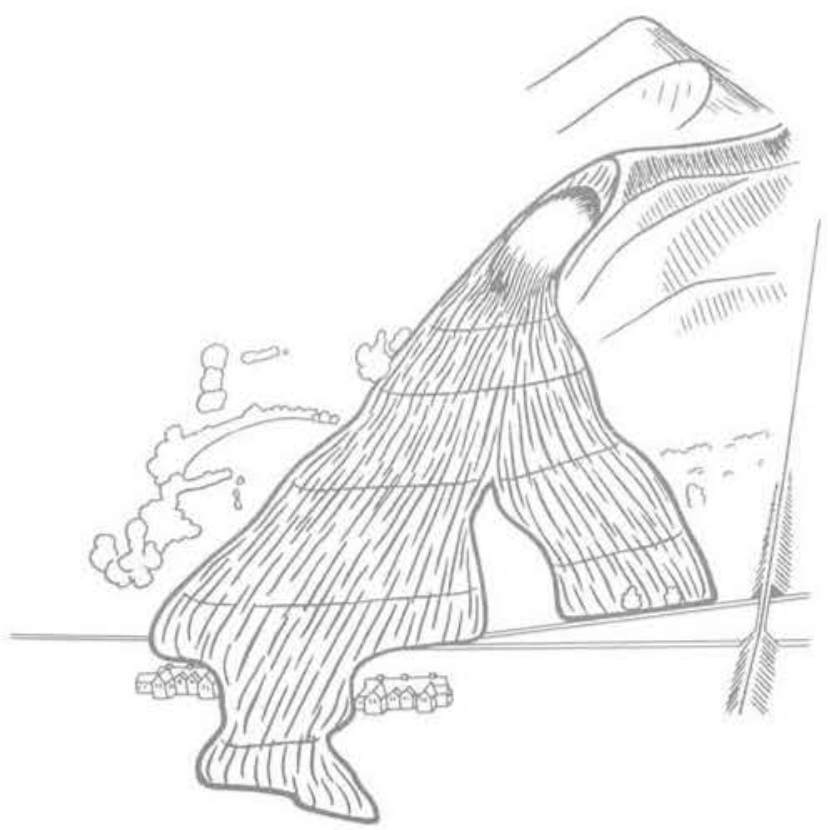

Fig. 23 Terrils d'Aberface. Vue générale (Bishop et al., 1969) 
Tableau IV

Calculs prévisionnels de stabilité:

hauteur à la rupture du remblai de Sallèdes selon diverses méthodes

\begin{tabular}{|c|c|c|c|}
\hline calcul & mesure & non circulaire & circulaire \\
\hline \multirow{2}{*}{$\begin{array}{c}\text { Contraintes } \\
\text { totales }\end{array}$} & $\begin{array}{c}\text { scissomètre } \\
\left(\mathrm{s}_{u}\right)\end{array}$ & $12 \mathrm{~m}$ & $16 \mathrm{~m}$ \\
\hline & $\begin{array}{c}\text { triaxial } \\
\left(\mathrm{C}_{u}\right)\end{array}$ & $6 \mathrm{~m}$ & $8 \mathrm{~m}$ \\
\hline \multirow{2}{*}{$\begin{array}{l}\text { Contraintes } \\
\text { effectives }\end{array}$} & $\begin{array}{l}\text { triaxial } \\
\left(c^{\prime}, \phi^{\prime}\right)\end{array}$ & - & $7-10 m$ \\
\hline & $\begin{array}{l}\text { boîte } \\
\left(c_{r}^{\prime}, \phi_{r}^{\prime}\right)\end{array}$ & $6 \mathrm{~m}$ & $4-7 \mathrm{~m}$ \\
\hline
\end{tabular}

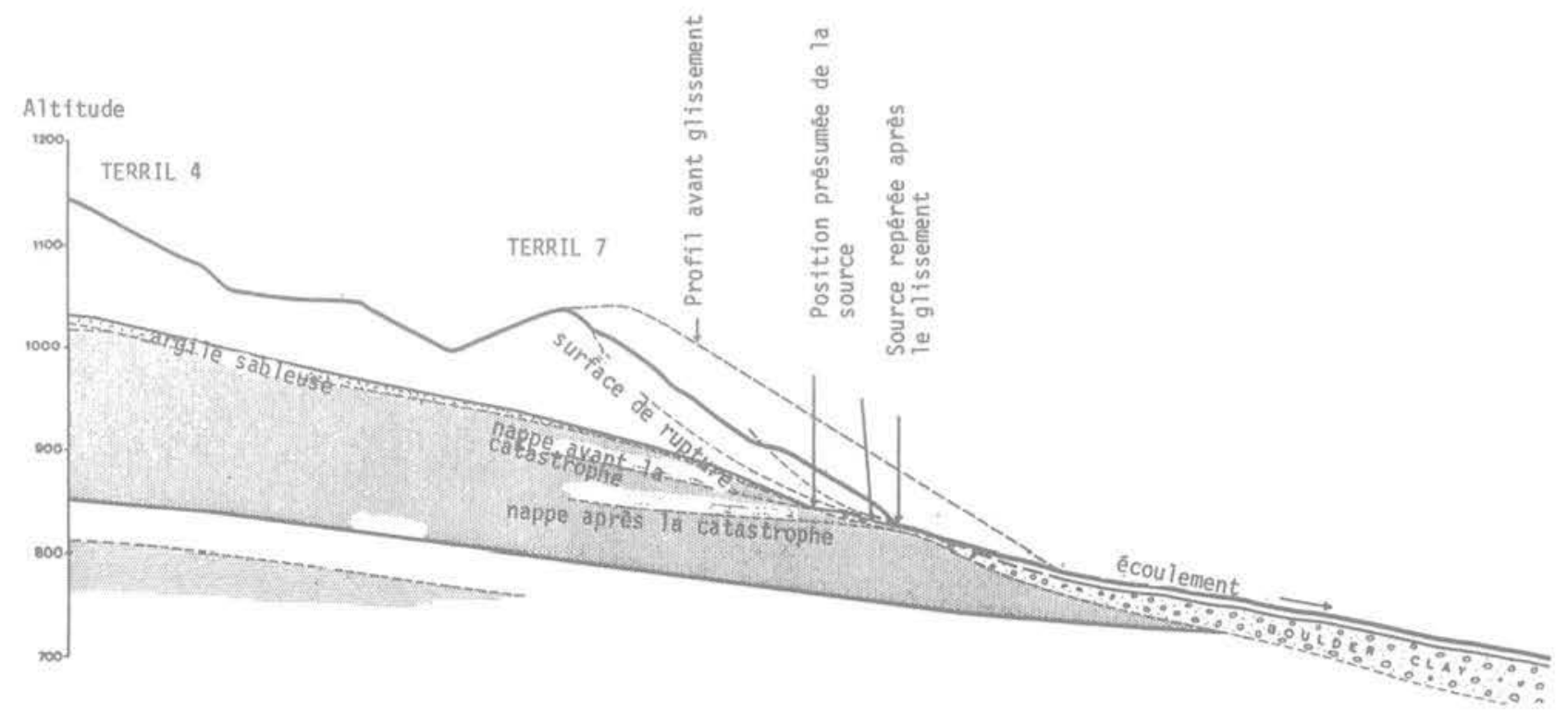

Fig. 24 Coupe de la rupture du terril $n^{\circ} 7 d^{\prime}$ Aberface (Bishop et al., 1969)

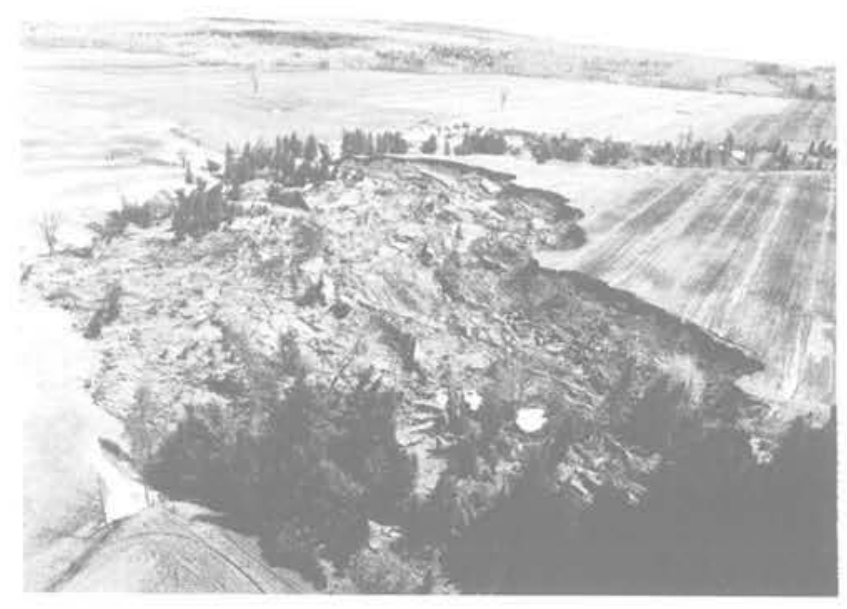

Fig. 25 Glissement du versant naturel en argile sensible lors d'un battage de pieux à Rigaud, Québec (Document Service Géotechnique MER)
Un brusque changement de régime hydraulique est considéré comme le facteur déclenchant du glissement :

- les mouvements de subsidence dûs à l'exploitation charbonnière ont créé de larges fissures de traction en amont des terrils : l'infiltration de l'eau y est très facile, l'écoulement se faisait aisément dans le grès, naturellement faillé, puis certainement fracturé par la subsidence:

- on a observé que la nappe fluctuait avec un faible temps de réponse lors de pluies abondantes;

- l'exhaure de la nappe est gênée en pied du terril $n^{\circ} 7$ par la couverture argileuse du grès : on avait d'ailleurs noté la présence d'une source en pied de talus peu avant le glissement.

On ajoutera que les calculs de stabilité réalisés au cours de l'enquête, s'appuyant sur des mesures de caractéristiques de résistance au cisaillement du matériau, et sur une position de nappe compatible avec les mesures réalisées après glissement et les observations faites avant glissement, ont correctement permis d'expliquer cette rupture. 


\subsubsection{Battage de pieux dans les versants argileux}

Le battage des pieux dans les formations argileuses se traduit par deux effets néfastes :

- transmission de vibrations:

- augmentation des pressions interstitielles lors du refoulement du sol.

Ces effets se sont révélées extrêmement nocifs à la stabilité des versants argileux (Massarsch, Broms, 1981) et de nombreux glissements importants se sont ainsi produits dans les argiles sensibles du Canada et de Scandinavie.

A titre d'illustration, la figure 25 montre le glissement survenu lors du battage de pieux destinés à supporter un pylone de ligne électrique.

\section{Conclusions}

L'expérience montre que les travaux de l'homme sont souvent générateurs de glissements de terrains et que, dans bien des cas (souvent les plus graves), ils résultent d'une aggravation imprévue d'une situation précaire de la stabilité; ceci a été évoqué dans quelques configurations classiques, mais on doit insister sur la gravité d'autres situations: il suffit de rappeler les questions qui se posent actuellement quant au rôle des grands aménagements de haute montagne dans la formation de coulées de boues très destructrices.

La connaissance actuelle du mécanisme des glissements de terrains est suffisamment avancée pour que des études intégrées (géologie, hydraulique, comportement des sols et des roches, mesures de déplacements, etc.) permettent de formuler un jugement valable sur une situation donnée et sur les perturbations attendues du fait des travaux envisagés. De ce fait, les lacunes qui demeurent (progressivité de la rupture, extension d'un glissement initial, par exemple) ou les méconnaissances locales (pressions interstitielles générées par une pluie de période de retour élevée) sont appelées à constituer des facteurs dont l'influence demeure dans l' "enveloppe de la sécurité "; à défaut d'analyses suffisantes, ces facteurs inattendus se cumulent aux facteurs cachés et deviennent le facteur déclenchant redouté.

Dans les circonstances oủ le doute demeure, il faut savoir instrumenter les sites en appareils de mesure; dans les cas les plus simples, les mesures serviront à confirmer les hypothèses, sinon elles permettront de faire un recalage du modèle et de revoir les jugements initiaux. Même dans les cas (et peut-être surtout) oủ les mesures infirment les hypothèses formulées a priori. les informations recueillies sont précieuses, puisqu'elles permettent au moins de prendre des mesures de sauvegarde propres à assurer la stabilité des constructions et la sécurité des vies.

\section{Références bibliographiques}

S. Amar, B. Gaudin, J. Legrand, M. Londez (1973). Franchissement de zones instables par des voies autoroutières. Comptes rendus. Symposium * Sol et sous-sol. Sécurité des constructions". Vol. 1, pp. 247271.

D. Beucler (1981). Glissement de la Mosquée «Emir Abd el Khader" à Constantine. Revue Française de Géotechnique, $n^{\circ}$ spécial 14 bis «Environnement et Géotechnique », pp. 190-193.
A.W. Bishop, L. Bjerrum (1960). The relevance of triaxial test to the solution of stability problems. Proc. Research conf. shear strength of cohesive soils. Boulder, pp. 437-501.

A.W. Bishop, J.N. Hutchinson, A.D. Penman, H.E. Ewans (1969). (1969). Geotechnical investigation into the causes and circumstances of the disaster of 21st october 1966. A selection of technical reports submitted to the Aberfan Tribunal. Welsh office, pp. 1-82.

L. Bjerrum (1972). Embankments on soft ground. Proc. ASCE specialty conf. on performance of earth and earth supported structures. Purdue University. Lafayette. Vol. 2, pp. 1-54.

L. Bjerrum (1973). Problems of soil mechanics and construction on soft clay and structurally instable soils. Comptes rendus $8^{\circ}$ congrès international mécanique des sols et travaux de fondations. Moscou. Vol. 3 , pp. 111-159.

J.-C. Blivet (1976). Stabilité des talus de déblais. Bulletin des Laboratoires des Ponts et Chaussées, $n^{\circ}$ spécial II "Déblais et remblais », pp. 9-22.

F. Blondeau, J.-C. Blivet, Y. Ung Seng (1976). Résistance au cisaillement des argiles raides. Bulletin des Laboratoires des Ponts et Chaussées, $n^{\circ}$ spécial III «Déblais et remblais", pp. 23-38.

F. Blondeau, D. Queyroi (1976). Rupture de la tranchée expérimentale de la Bosse Galin. Bulletin des Laboratoires des Ponts et Chaussées, $n^{\circ}$ spécial III $\propto$ Déblais et remblais ", pp. 59-69.

F. Blondeau, M. Virollet (1976). Comportement des murs de soutènement en zone instable. Bulletin des Laboratoires des Ponts et Chaussés, $n^{\circ}$ spécial II "Versants naturels", pp. 149-154.

F. Blondeau, A. Perrot, G. Pilot (1977). Étude en vraie grandeur de versants naturels instables. $9^{\circ}$ congrès international de mécanique des sols et travaux de fondations. Tokyo. Vol. 2, pp. 21-24.

F. Blondeau, P. Morin (1980). Rupture d'un remblai expérimental sur versant à Sallèdes. Bulletin des Laboratoires des Ponts et Chaussées, $n^{\circ} 106$, pp. 133137.

J.-P. Flotte (1981). Mouvements liés à des travaux de défense et de restauration des sols. Revue Française de Géotechnique, $n^{\circ}$ spécial 14 bis « Environnement et géotechnique n, pp. 194-197.

J.-P. Gosset, J.-P. Khizardjian (1976). Étude de la tranchée d'essai du Tronchon sur l'autoroute A6. Bulletin des Laboratoires des Ponts et Chaussées, $n^{\circ}$ spécial III "Déblais et remblais", pp. 49-58.

J. Huder, R. Duerst (1981). Safety consideration for cut in unstable slope. Comptes rendus, $10^{\circ}$ congrès international de mécanique des sols et des travaux de fondations. Stokholm. Vol. 3, pp. 431-436.

R. Japelli, A. Musso (1981). Slope response to reservoir water level fluctuations. Comptes rendus, $10^{\circ}$ congrès international de mécanique des sols et des travaux de fondations. Stockholm. Vol. 3, pp. 437-442.

A. Jeannette, A. Millies-Lacroix (1965). La lutte contre l'érosion dans le domaine rifain. Le rôle des banquettes sur la stabilité des versants. Mines et géologie. Rabat, $n^{\circ} 23, p p .49-56$.

B.J. John-St, G.F. Sowers, C.E. Weaver (1969). Slickensides in residual soils and there engineering significance. Comptes rendus, $7^{\circ}$ congrès IMSTF Mexico. Vol. 2, pp. 591-557. 
J. Krahn, R.F. Johnson, D.G. Fredlund, Clifton (1979). A highway cut failure in cretaceous sediments at Maymont, Saskatchewan. Revue Canadienne de Géotechnique. Vol. 16, $n^{\circ} 4$, pp. 703-733.

P. La Rochelle, R.J. Marsal (1981). Slope stability. General report. X Int. conference soil mechanic foundation engineering. Stockholm. Tirage préliminaire, pp. 141-161.

S. Lerouil F. Tavenas, C. Mieussens, M. Peignaud (1978). Construction pore pressures in clay foundations under embankments; part. 2. Generalized behaviour. Revue canadienne de géotechnique. Vol. 15.1, pp. 66-82 et Bulletin des Laboratoires des Ponts et Chaussées, $n^{\circ} 98, p p .53-67$ (en français).

G. Lheriteau, M. Moudden, G. Post (1977). Étude de la stabilité des rives de la cuvette du barrage ldriss $1^{\text {er }}$ au Maroc. Revue Française de Géotechnique $n^{\circ} 1$, pp. 7-16.

K.R. Massarsch, B.B. Broms (1981). Pile driving in clay slopes. Comptes rendus $10^{\circ}$ congrès international de mécanique des sols et des travaux de fondations. Stockholm. Vol. 3, pp. 469-474.

A. Mayer, P. Habib (1961). Étude de la stabilité de deux crassiers d'usine métallurgiques. Comptes rendus. $5^{\mathrm{e}}$ congrès international de mécanique des sols et des travaux de fondations. Paris. Vol. II, pp. 669-672.

L.R. Moore (1969). Geological report on the tipping site and its environs at Merthyr Vale and Aberfan. A selection of technical reports submitted to the Aberfan Tribunal. Welsh office, pp. 147-185.

N. Morgenstern, G.E. Blight, N. Janu, D. Resendiz (1977). Slopes and excavations. $9^{\circ}$ congrès international mécanique des sols et travaux de fondations. Tokyo. Vol. 2, pp. 547-604.

G. Pilot (1969), Discussions. $7^{\circ}$ congrès international de mécanique des sols et travaux de fondations. Mexico. Vol. III, pp. 408-409.

G. Pilot (1972). Study of five embankment failures on soft soils. Proc. ASCE specialty conf. on performance of earth and earth supported structures. Purdue University. Lafayette. Vol. I, part. 1, pp. 81-100.

G. Pilot (1976). La stabilité des remblais sur sols mous. Bulletin des Laboraoires des Ponts et Chaussées, $n^{\circ}$ spécial III «Stabilité des talus", pp. 83-106.

G. Pilot (1976). La stabilité des remblais sur sols mous. Bulletin des Laboratoires des Ponts et Chaussées, $\mathrm{n}^{\circ}$ spécial II " Déblais et remblais", pp. 70-82.

G. Pilot, B. Pincent, G. Cartier, F. Blondeau (1979). Mesure des déplacements et confortement des glissements de remblais sur versants. $7^{\circ}$ congrès européen de mécanique des sols et travaux de fondations. Brighton. Vol. 3, pp. 253-260.

G. Pilot, B. Trak, P. La Rochelle (1982). Calcul en contraintes effectives de la stabilité des remblais sur sols mous. A paraître.

G. Sanglerat (1978). Pathologie des fondations et reprises en sous-œuvre. CAST Lyon, $44 \mathrm{p}$.

C. Schroeder (1981). Exploitation rationnelle d'un terril. Compte rendu des secondes journées nationales de géotechnique. Nantes. Revue Française de technique, $n^{\circ} 14$ bis, pp. 80-84.

A. Silleran, (1981). Glissements de terrain liés à des travaux. Revue Française de Géotechnique, $n^{\circ}$ spécial 14 bis *Environnement et Géotechnique", pp. 198202.

V. Silvestri (1980). The long term stability of a cutting slope in an overconsolidaled sensitive clay. Revue Canadienne de Géotechnique. Vol. 17, n³, pp. 337351.

A.W. Skempton, P. La Rochelle (1965). The Bradwell slip. A short term failure in London clay. Geotechnique. Vol. XV.3, pp. 221-242.

A.W. Skempton (1964). Long term stability of clay slopes. Geotechnique 14, pp. 77-101.

A.W. Skempton (1977). Slope stability of cuttings in brown London clay. Conference au $9^{\circ}$ congrès IMSTF de Tokyo. Vol. 3, pp. 261-270.

A.W. Skempton, J. Hutchinson (1969). Stability of natural slopes and embankment foundations. État des connaissances. Comptes rendus $7^{\circ}$ congrès international de mécanique des sols et travaux de fondations. Mexico, pp. 291-340.

F. Tavenas, S. Le Roueil (1980). The behaviour of embankments on clay foundations. Revue Canadienne de Géotechnique, $n^{\circ} 17$, pp. 236-260.

Y.B. Trzhtzinskii (1978). Landslides along the Angara reservoirs. Bulletin AIGI, $n^{\circ} 17$, pp. $42-43$.

P.R. Vaughan, H.J. Walbrancke (1973). Pore pressure changes and the delayed failure of cutting slopes in overconsolidated clay. Geotechnique 23, pp. 531-539.

H.J. Walbrancke (1976). Pore pressures in clay embankments and cuttings. Ph.D. Thesis. University of London.

A.W. Woodland (1969). Geological report on the Aberfan tip disaster of october 21 st 1966. A selection of technical reports submitted of the Aberfan Tribunal. Welsh office, pp. 119-145.

Q. Zaruba, V. Mencl (1969). Landslides and there control. Elsevier-Academica, 205 p. 
